\title{
Designed hydrocolloid interpenetrating polymeric networks for clinical applications of novel drug-carrying matrix systems using Tris (6-isocyanatohexyl) isocyanurate and hydroxypropylmethylcellulose
}

\author{
Hsia-Wei Liu ${ }^{\mathrm{a}}$, Jen-Ray Chaw ${ }^{\mathrm{b}}$, Yu-Chao Shih ${ }^{\mathrm{c}}$ and Ching-Cheng Huang ${ }^{\mathrm{d}, \mathrm{e},{ }^{*}}$ \\ ${ }^{a}$ Department of Life Science, Fu Jen Catholic University, New Taipei City 24205, Taiwan \\ ${ }^{b}$ Graduate Institute of Applied Science and Engineering, Fu Jen Catholic University, New Taipei City \\ 24205, Taiwan \\ ${ }^{c}$ Department of Materials Science and Engineering, National Tsing-Hua University, Hsinchu 30013, \\ Taiwan \\ ${ }^{d}$ Department of Biomedical Engineering, Ming-Chuan University, 5 De Ming Rd., Gui Shan District, \\ Taoyuan, 333, Taiwan \\ ${ }^{e}$ Metal Industries Research \& Development Centre, 6F, No.162-24, Sec.3, Hsin-Yi Rd., Taipei 10658, \\ Taiwan
}

\begin{abstract}
Hydroxypropyl methylcellulose (HPMC) was employed in this study to design controllable drug release systems because of its non-toxic nature, swelling properties. New interpenetrating polymer networks (IPN) of HPMC / tri-isocyanate crosslinked polyurethane (TCPU) could be prepared on the surfaces of IPN materials. To design "Novel Drug-carrying Matrix Systems", incorporation of novel structure is important to the possible formation of drug-carrying spaces within the material, which was achieved by using Tris (6-isocyanatohexyl) isocyanurate with three soft hexyl arms in this study. A series of novel drug-carrying matrix systems prepared by crosslinking reaction could be candidates for an excellent and smart potential material. When the polymeric networks were established on the surfaces of resulting materials, the developed hydrophilic interpenetrating polymeric structures of HPMC/ polyurethane could provide good wettability to the wound dressings, particularly for moisture healing application. The materials containing HPMC/polyurethane networks using $1 \%$ cross-linking agent showed a water uptake value of $5.1 \%$ after one hour, which has great potential for use as wound dressings for moisture healing. Furthermore, a new drug delivery system of hydrophilic IPN was successfully designed and established.
\end{abstract}

Keywords: Interpenetrating polymer network, hydroxypropyl methylcellulose, drug delivery

\footnotetext{
${ }^{*}$ Corresponding author: Ching-Cheng Huang, Department of Biomedical Engineering, Ming-Chuan University, 5 De Ming Rd., Gui Shan District, Taoyuan, 333, Taiwan. Tel.: +886-988-915-063; Fax: +886-3-359-3840; E-mail: jcchuang@mail.mirdc.org.tw.
} 


\section{Introduction}

Review of biomedical application, the clinical demands of drug carrying and wound management systems is critical. The way for rapid and proper healing is necessary in the treatment of wounds such as severe burns, trauma, diabetic and venous stasis ulcers, and similar tissue damages. The healing response of tissues would involve a clinical complex interaction between cell and tissue, extracellular matrix molecules, and some soluble mediators. Sometimes, such complex process would be simplified by dividing the healing response into several categories that agree with the temporal sequence of normal healing: homeostasis, inflammation, proliferation, and remodeling. In cases of severe and massive amounts of skin damage, or in the presence of complicated and non-healing wounds, immediate coverage of the wound surface with a dressing is necessary. For the clinical demand of wound healing, a suitable material or design for dressing must achieve the functions of the natural skin by protecting the area from loss of fluid and proteins, preventing infection from bacterial invasion, and subsequent tissue damage. Therefore, numerous skin replacements are either currently available for clinical need or in clinical testing. These include temporary and permanent skin replacements, epidermal and dermal skin replacements, and synthetic/biosynthetic and biologic skin replacements. Numerous materials have been proposed, modified and used for biomedical applications. Synthetic materials, such as polyurethane, polyelectrolyte, polyimide, polyamide, polyacrylate, polynorborene, polycarbonate, hydroxyapatite, poly (lactide-co-glycolide), and polylactide, have been applied either alone or in combination with naturally derived materials including alginate, chitosan, collagen, silk fibroin, starch and cellulose [1-24]. Furthermore, new wound dressing or drug release carrier could be designed and prepared to obtain new functions. Also the specific structure could be designed for different applications. Specifically, hydroxypropyl methylcellulose (HPMC) was used to control drug release from several pharmaceutical systems because of its non-toxic nature, swelling properties. Review of drug release from hydrophilic drug carrying matrix systems indicated that erosion is an important aspect for both poorly soluble and well soluble drugs [25]. Furthermore, fundamental principles of polymer chemistry and dissolution mechanisms are important in the development and manufacturing of hydrophilic drug carrying matrix systems with consistent dissolution performance [25]. That is, incorporation of HPMC into the drug carrying matrix systems would provide a drug controllable factor for the matrix systems.

Usually, the cross-linking agent would be a good potential way to create new functional properties of the polymeric material, which was employed in this study to create new drug-carrying spaces within collapsed polymeric material. To achieve the aim of "Novel Drug-carrying Matrix Systems", incorporation of novel structure is of critical importance in possible formation of drug-carrying spaces within material using Tris (6-isocyanatohexyl) isocyanurate (Tri-isocyanate with three soft hexyl arms). A series of novel drug-carrying matrix systems would be prepared by using crosslinking reaction of tris (6-isocyanatohexyl) isocyanurate and incorporation of HPMC. The resulting drug-carrying matrix systems could be candidates for an excellent and smart potential material.

\section{Experimental}

\subsection{Materials}

- HPMC was purchased from Sigma-Aldrich. 
(A)

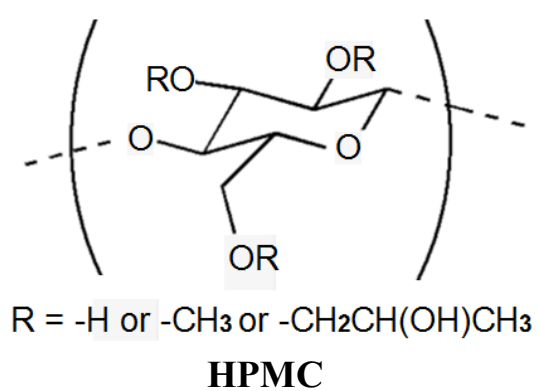

(B)

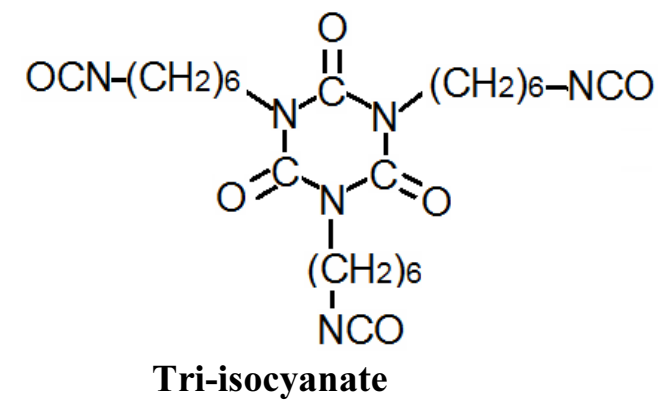

Schematic 1. Structures of (A) Hydroxypropyl methylcellulose (HPMC) and (B) Tri (6-isocyanatohexyl) isocyanurate (Tri-isocyanate).

- Tris (6-isocyanatohexyl) isocyanurate (Tri-isocyanate, Trade name TOLONATE, Rhone-Poulene Co., Texas)-a tri-NCO terminated crosslinker with $\mathrm{Mw}=504.6$ and NCO-equivalent weight 183 . Its chemical structure is shown below (see Schematic 1).

- The acrylate-base adhesives (medical grade) were purchased from Chensin Packing Industry Co. Ltd. (Taiwan) which is a manufactory for preparing medical tape and dressing. After reactions, the biology evaluation following ISO 10993 could be employed to check toxicity of resulting materials.

\subsection{Preparations of interpenetrating polymeric networks of hydroxypropyl methylcellulose/acrylate-} base adhesives (HPMC/PAc IPN)

The acrylate-base adhesives (purchased from Chensin Packing Industry Co., Ltd., Taiwan) were mixed with HPMC at room temperature to obtain high viscous HMPC/adhesives. The resulting HPMC/PAc IPN was heated and mixed at $60^{\circ} \mathrm{C}$ for 1 hour. After mixing, methanol was employed to wash the resulting HPMC/PAc IPN materials for 12 hours.

\subsection{Preparations of interpenetrating polymeric networks of hydroxypropyl methylcellulose/tri- isocynate crosslinked polyurethane (TCPU/HPMC/PAc IPN)}

The polyacrylate-base adhesives were mixed with HPMC at room temperature to obtain high viscous HPMC/PAc IPN), after adding the crosslinking agent (tri-isocynate), the resulted HPMC/PAc IPN was heated at $60^{\circ} \mathrm{C}$ for 1 hour. At the same time, the cross-linking reaction was carried and a series of interpenetrating polymeric networks of polyacrylate/hydroxypropyl methylcellulose/tri-isocynate crosslinked polyurethane (TCPU/HPMC/PAc IPN) were obtained. The resulting TCPU/HPMC/PAc IPN could be characterized by using FTIR and the disappearance of the NCO group and the appearance of $\mathrm{N}-\mathrm{H}$ group could be observed. After cross-linking reaction, methanol was employed to wash the resulting IPN materials for 12 hours and methanol reacted with residual -NCO groups of tris (6isocyanatohexyl) isocyanurate. The toxicity of tris (6-isocyanatohexyl) isocyanurate could be avoided. 
Table 1

New designed hydrocolloid drug-carrying matrix systems containing IPN

\begin{tabular}{lll}
\hline & HPMC/PAc IPN & $:$ \\
\hline HPMC/PAc IPN & 100 & 0 \\
TCPU/HPMC/PAc IPN1 & 100 & 1 \\
TCPU/HPMC/PAc IPN2 & 100 & 10 \\
\hline Note: a) The ratios between HPMC/PAc IPN and trimer (TOLONATE) are calculated by weight $\%$.
\end{tabular}

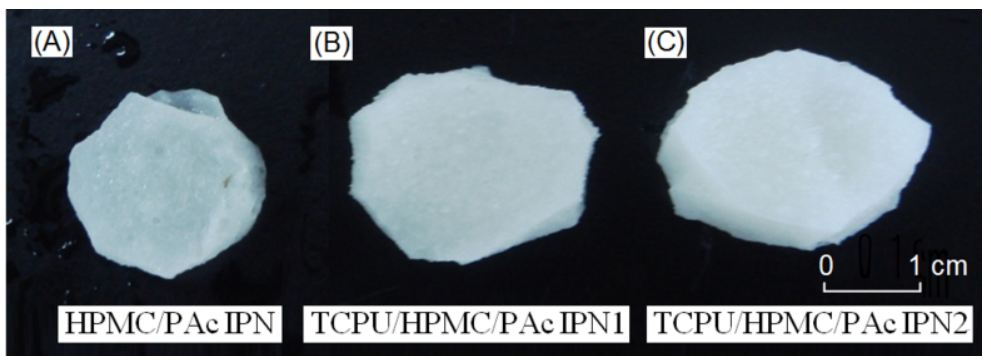

Fig. 1. New hydrocolloid drug-carrying matrix systems containing interpenetrating polymeric networks (IPN).

\section{Results and discussion}

\subsection{Clinical application for new wound dressings containing interpenetrating polymeric networks of} polyurethane /hydroxypropyl methylcellulose/polyacrylate-base adhesives (TCPU/HPMC/PAc IPN)

New wound dressings containing TCPU/HPMC/PAc IPN were made with various amounts of Trimer (see Table 1 and Figure 1). Infrared radiation (IR) spectroscopy could be obtained from the cast film. This analysis was employed to check the end of cross-linking reaction, verifying the disappearance of the NCO group at $2266 \mathrm{~cm}^{-1}$ and the appearance of N-H group at $3000-3400 \mathrm{~cm}^{-1}$. The resulting TCPU/HPMC/PAc IPN also display a large absorption peak at $1735 \mathrm{~cm}^{-1}$ of IR spectrum corresponding to the $\mathrm{C}(\mathrm{O})-\mathrm{O}$ ester linkage in the polyurethane(PU) segment. Presence of expected peaks implies that the reaction was completed and the PU segment was formed. An absorption band of the $\mathrm{N}-\mathrm{H}$ stretching mode at $3290 \mathrm{~cm}^{-1}$ was observed. These vibrations are strong evidence for the formation of flexible PU segments after cross-linking reaction and end of cross-linking reaction. Furthermore, the end of cross-linking reaction would hint the incorporation of flexible property into the resulting materials such as TCPU/HPMC/PAc IPN. The flexibility of TCPU/HPMC/PAc IPN might be higher than that of HPMC/PAc IPN because of hydrogen bonding. It might be due to the formation of the new interpenetrating polymeric networks containing flexible TCPU units, which were confirmed by IR results. Formation of interpenetrating polymeric networks hints the three-dimensional structure of the resulting matrix. When the polymeric systems were established on the surfaces of wound dressings, the advanced hydrophilic interpenetrating polymeric structures of HPMC/PAc IPN could provide good wettability to match the clinical demand of wound dressings. However, the structures of HPMC/PAc IPN would be collapsed after 250 hours because of weak matrix structure (see Figure 2). The uptake values of the resulting materials were observed during the 250 hours because of the collapsed structure of HPMC/PAc IPN after 250 hours (see Figure 2). The wound dressings containing TCPU/HPMC/PAc IPN1 using 1\% cross-linking agent still show good wettability to the wound dressings and a water uptake value of 5.1\% after one hour (see Figure 2). Furthermore, drug could be con- 
sidered and/or incorporated into the hydrophilic polymeric systems of polyurethane/Hydroxypropyl methylcellulose/polyacrylate-base adhesives structure. Selected drugs within hydrophilic TCPU/HPMC/PAc IPN could be released and monitored by high performance liquid chromatography.

\subsection{Potential controllable drug delivery matrix systems}

This paper deals with the physical description and designed matrix systems of drug release. In the study, matrix systems are considered in the broad frame of the controlled release systems and the concept of IPN structure is briefly discussed. In general, drug release applications of the designed matrix system are considered with particular emphasis on the essential factors ruling the release kinetics, such as matrix swelling, drug dissolution, drug diffusion, drug - polymer interaction, initial drug distribution and particle size distribution [25]. The cross-linking reaction could enhance the strength of construction, which could maintain some space within the construction and provide good potential application for controllable drug delivery matrix systems. The linkage derived from crosslinked reaction could be applied to be a key controllable unit, which is why tris (6-isocyanatohexyl) isocyanurate was employed in this study. Three soft hexyl arms of tris (6-isocyanatohexyl) isocyanurate could provide enough space for drug charring.

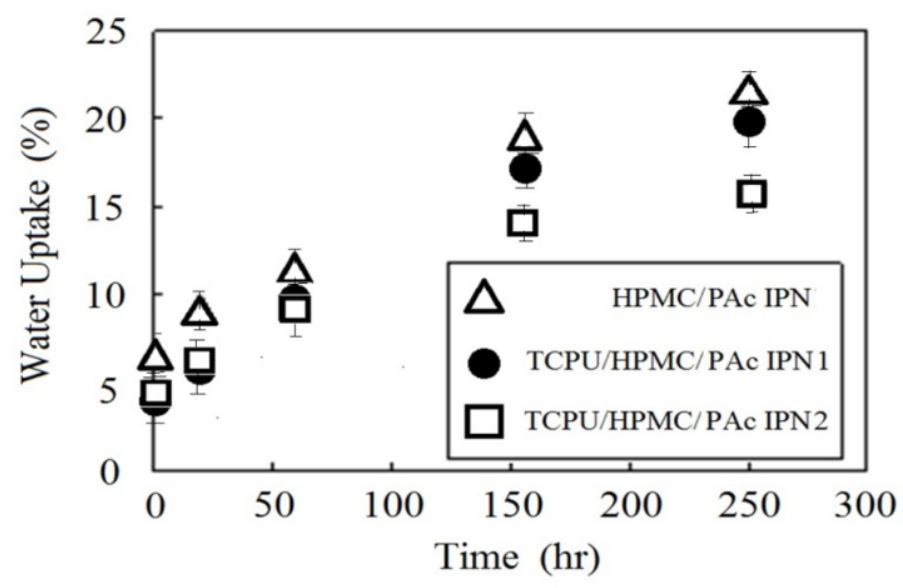

Fig. 2. Water uptake of new hydrocolloid interpenetrating polymeric networks (IPN).
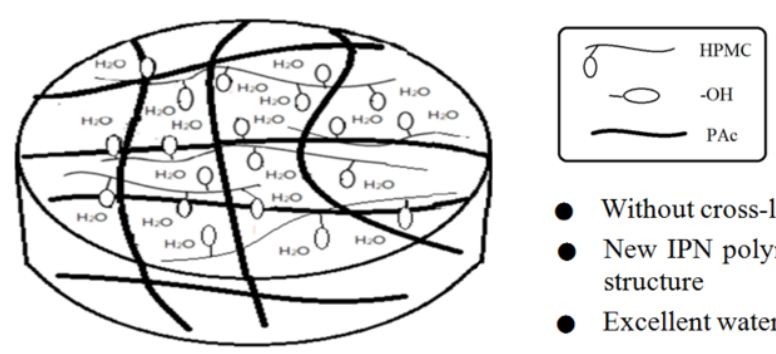

- Without cross-linking agents

- New IPN polymeric network without cross-linked structure

- Excellent water absorption

Fig. 3. Proposed Model of New hydrocolloid drug-carrying matrix systems containing interpenetrating polymeric networks (IPN) of hydroxypropyl methylcellulose (HPMC/PAc IPN). 

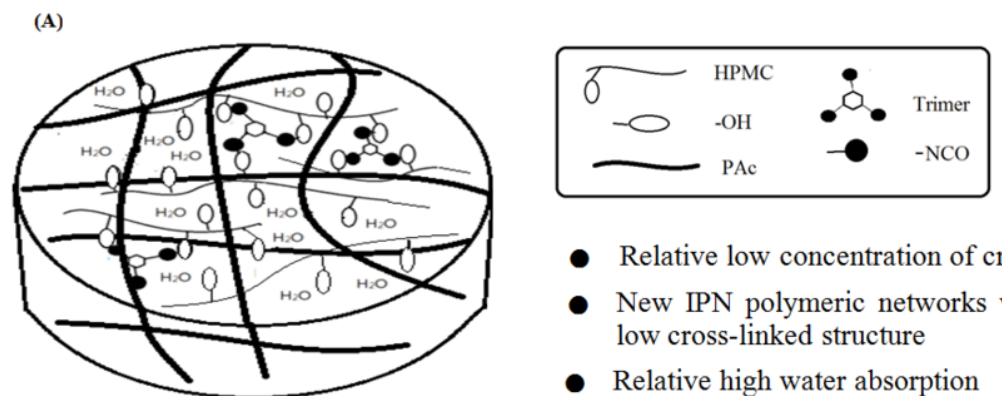

- Relative low concentration of cross-linking agents

- New IPN polymeric networks with corresponding low cross-linked structure

- Relative high water absorption
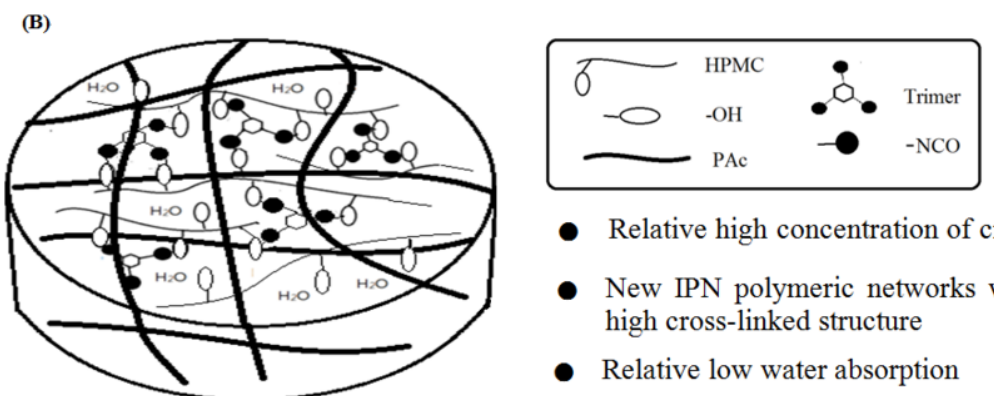

- Relative high concentration of cross-linking agents

- New IPN polymeric networks with corresponding high cross-linked structure

- Relative low water absorption

Fig. 4. Proposed model of new hydrocolloid interpenetrating polymeric networks containing tri-isocyanate crosslinked polyurethane, hydroxypropyl methylcellulose and polyacrylate adhesives with various amounts of cross-linking agents (trimer) such as (A) TCPU/HPMC/PAc IPN1 and (B) TCPU/HPMC/PAc IPN2).

\subsection{Schematic models of new hydrocolloid drug-carrying matrix systems derived from HPMC/PAc IPN for controllable drug delivery}

Schematic model of new hydrocolloid drug-carrying matrix systems without cross-linking agents (trimer) derived from HPMC/PAc IPN is exhibited in Figure 3. New hydrocolloid drug-carrying matrix systems without trimers (HPMC/PAc IPN) show excellent water adsorption which is due to the high content of hydroxyl units $(-\mathrm{OH})$ within the new IPN matrix. Furthermore, schematic models of new hydrocolloid drug-carrying matrix systems containing interpenetrating polymeric networks of triisocyanate crosslinked polyurethane, hydroxypropyl methylcellulose and polyacrylate adhesives (such as TCPU/HPMC/PAc IPN1 and TCPU/HPMC/PAc IPN2) with various amounts of cross-linking agents are exhibited in Figure 4. The tri-isocyanate crosslinked polyurethane provides the crosslinking structure within the IPN materials and flexible property of the IPN materials containing urethane groups. However, the formation of tri-isocyanate crosslinked polyurethane within the IPN materials would decrease hydrophilic hydroxyl units within the IPN materials (TCPU/HPMC/PAc IPN). The water uptake of the TCPU/HPMC/PAc IPN materials is lower than that of HPMC/PAc IPN. Different amounts of trimer will react with the hydroxyl group of various contents, which can affect the water absorption ability of the TCPU/HPMC/PAc IPN materials. Relative low concentration of crosslinking agents was applied in preparation of TCPU/HPMC/PAc IPN1 and new drug release IPN matrix with relative low cross-linked structure was obtained (see Figure 4(A)). TCPU/HPMC/PAc IPN1 shows relative high water absorption ability because of relative high contents of hydroxyl groups. Relatively, relative high concentration of cross-linking agents was employed in preparation of TCPU/HPMC/PAc IPN2 and new drug release IPN matrix with corresponding high cross-linked structure was obtained (see Figure 4(B)). 


\section{Conclusion}

New drug delivery system of hydrophilic IPN was successfully designed and installed. HPMC was used to control drug release from several pharmaceutical systems because of its functional hydroxyl groups, non-toxic nature, swelling properties. In this study, a new designed TCPU/HPMC/PAc IPN could also be prepared on the surfaces of wound dressings. When the polymeric systems were established on the surfaces of wound dressings, the advanced hydrophilic interpenetrating polymeric structures of TCPU/HPMC/PAc IPN could provide good wettability, which contributes to its application as wound dressings and creates a good hydrophilic microenvironment. The wound dressings containing hydroxypropyl methylcellulose/polyurethane networks using $1 \%$ cross-linking agent show a water uptake value of $5.1 \%$ after one hour. For the clinical demand of drug carrying matrix system, drug could be considered and/or incorporated into the hydrophilic HMPC/TCPU IPN matrix structure. New drug carry and delivery systems of hydrophilic IPN were also successfully created and installed. The designed matrix systems could produce a controllable potential for drug delivery, because of physical interpenetrating polymeric networks and chemical urethane groups.

\section{Acknowledgement}

The authors gratefully acknowledge Well Lands Enterprise Co., Ltd. and Ministry of Economic Affairs, Taiwan for their financial supports.

\section{References}

[1] S. Ito, M. Hashimotob, B. Wadgaonkar, N. Svizero, R.M. Carvalho, C. Yiu, F.A. Rueggeberg, S. Foulger, T. Saito, Y. Nishitanii, M. Yoshiyamai, F.R. Tay and D.H. Pashley, Effects of resin hydrophilicity on water sorption and changes in modulus of elasticity, Biomaterials 26 (2005), 6449-6459.

[2] D.J. Liaw, C.C. Huang and B.Y. Liaw, Synthesis and properties of polyurethanes based on bisphenol-S derivatives, Polymer 39 (1998), 3529-3535.

[3] D.J. Liaw, C.C. Huang, H.C. Sang and E.T. Kang, Intramolecular hydrophobic aggregation of amphiphilic polysulfobetaine with various hydrophobic groups in aqueous solution, Langmuir 15 (1999), 5204-5211.

[4] D.J. Liaw, C.C. Huang, H.C. Sang and P.L. Wu, Macromolecular microstructure, reactivity ratio and viscometric studies of water-soluble cationic and/or zwitterionic copolymers, Polymer 41 (2000), 6123-6131.

[5] D.J. Liaw and C.C. Huang, Synthesis and characterization of the poly (sulfobetaine)s and the corresponding cationic polymers derived from N,N'-Dialkylamino phenyl methacrylamide macromol, Chem. Phys. 201 (2000), 1101-1107.

[6] D.J. Liaw, T.P. Chen and C.C. Huang, Novel active ester-bridged copolynorbornene materials containing terminal functional hydroxyl, amino, methacryloyl or ammonium groups via ring-opening metathesis polymerization, J. Polym. Sci., Part A: Polym. Chem. 43 (2005), 4233-4247.

[7] D.J. Liaw, T.P. Chen and C.C. Huang, Self-assembly aggregation of highly stable copolynorbornenes with am-phiphilic architecture via ring-opening metathesis polymerization, Macromolecules 38 (2005), 3533-3538.

[8] G. Zhai, S.C. Toh, W.L. Tan, E.T. Kang, K.G. Neoh, C.C. Huang and D.J. Liaw, Poly (vinylidene fluoride) with grafted zwitterionic polymer side chains for electrolyte-responsive microfiltration membranes, Langmuir 19 (2003), 7030-7037.

[9] D.J. Liaw, C.C. Huang and W.H. Chen, Color lightness and highly organosoluble polyamides, polyimides and poly(amide-imide)s based on noncoplanar 2,2'-dimethyl-4,4'-biphenylene units, Polymer 47 (2006), 2337-2348.

[10] D.J. Liaw, C.C. Huang and E.T. Kang, Novel fluorescent polynorbornenes with multi-functional armed structure by using highly stable block macroinitiators via a combination of living ring-opening metathesis polymerization and atom transfer radical polymerization, Polymer 37 (2006), 3057-3064.

[11] D.J. Liaw, C.C. Huang, S.M. Hong, W.H. Chen, K.R. Lee and J.Y. Lai, Molecular architecture effect on active structure of polynorbornenes with pendant $\alpha, \beta$-unsaturated amide or ester bridged chains via ring-opening metathesis polymerization, Polymer 47 (2006), 4613-4621. 
[12] T. Katsumata, M. Maitani, C.C. Huang, M. Shiotsuki and T. Masuda, Synthesis and properties of various poly (diphenylacetylenes) containing tert-amine moieties, Polymer 49 (2008), 2808-2816.

[13] D.J. Liaw, C.C. Huang and S.M. Hong, Novel doubly polymerizable functional norbornene: dynthesis, reactivity and its macromolecular architectures from dual cure via ring-opening metathesis polymerization and radical photopolymerization, J. Polym. Sci., Part A: Polym. Chem. 44 (2006), 6287-6298.

[14] C.C. Huang and G.W. Chang, Designed nerve conduits derived from chitosan-coated alginate layers, PMSE Preprints Am. Chem. Soc. Div. Polym. Mater. Sci. Eng. 101 (2009), 465.

[15] D.J. Liaw, C.C. Huang and E.T. Kang, Effect of architecture and environments on polymeric molecular assemblies of novel amphiphilic diblock copolynorbornenes with narrow polydispersity via living ring-opening metathesis polymerization (ROMP), J. Polym. Sci., Part A: Polym. Chem. 44 (2006), 2901-2911.

[16] C. Silve, E. Lopez, B. Vidal and D.C. Smith, Nacre initiates biomineralization by human osteoblasts maintained in vitro, Calcif. Tissue Int. 51 (1992), 363-369.

[17] E.T. Kang, K.G. Neoh, W. Chen, K.L. Tan, D.J. Liaw and C.C. Huang, Surface structures and adhesion characteristics of poly (tetrafluoroethylene) films after modification by graft copolymerization, J. Adhesion Sci. Technol. 10 (1996), $725-743$.

[18] Z.F. Li, E.T. Kang, K.G. Neoh, K.L. Tan, C.C. Huang and D.J. Liaw, Surface structures and adhesive-free adhesion characteristics of polyaniline films after modification by graft copolymerization, Macromolecules 30 (1997), 33543362.

[19] W. Chen, K.G. Neoh, E.T. Kang, K.L. Tan, D.J. Liaw and C.C. Huang, Surface modification and adhesion characteristics of polycarbonate films after graft copolymerization, J. Polym. Sci., Part A: Polym. Chem. 36 (1998), 357-366.

[20] G. Zhai, S.C. Toh, W.L. Tan, E.T. Kang, K.G. Neoh, C.C. Huang and D.J. Liaw, Poly (vinylidene fluo-ride) with grafted zwitterionic polymer side chains for electrolyte-responsive microfiltration membranes, Langmuir 19 (2003), 7030-7037.

[21] G. Atlan, O. Delatre, S. Berland, A. Le Faou, G. Nabias, D. Cot and E. Lopez, Interface between bone and nacre implants in sheep, Biomaterals 20 (1997), 1017-1022.

[22] J.E. Barralet, L. Grover, T. Gaunt, A.J. Wright, I.R. Gibson, Preparation of macroporous calcium phosphate cement tissue engineering scaffold, Biomaterials 23 (2002), 3063-3072.

[23] J.M. Karp, M.S. Shoichet and J.E. Davies, Bone formation on two dimensional poly(DL-lactide-co-glycolide) (PLGA) films and three dimensional PLG Shoichet, A tissue engineering scaffolds in vitro, J. Biomed. Mater. Res. A 64 (2003), 388-396.

[24] J.A. Roether, J.E. Gough, A.R. Boccaccini, L.L. Hench, V. Maquet and R. Jerome, Novel bioresorbable and bioactive composites based on bioactive glass and polylactide foams for bone tissue engineering, J. Mater. Sci. Mater. Med. 13 (2002), 1207-1214.

[25] D. Zhou, D. Law, J. Reynolds, L. Davis, C. Smith, J. L. Torres, V. Dave, N. Gopinathan, D.T. Hernandez, M.K. Springman and C.C. Zhou, Understanding and managing the impact of HPMC variability on drug release from controlled release formulations, Journal of Pharmaceutical Sciences 103 (2014), 1664-1672 